\title{
DETERMINANTES DA INFORMALIDADE E DESEMPREGO NO MERCADO DE TRABALHO DA REGIÃO METROPOLITANA DO RECIFE NO PERÍODO DE 2010
}

Leandro Batista Duarte'.

1 - Professor do Departamento de Ciências Sociais Aplicada - DCIS da Universidade Estadual de Feira de Santana - UEFS. Doutor em Economia pela Universidade Federal de Pernambuco (UFPE). Mestre em Economia pela Universidade Federal de Viçosa (UFV). Graduação em Economia pela Universidade Estadual de Santa Cruz (UESC). E-mail: leandro.duarte1@hotmail.com

\section{Resumo}

Diante da importância de compreender melhor o mercado de trabalho, o presente estudo avalia o comportamento dos determinantes da informalidade e desemprego através de um modelo econométrico que estima as chances de um individuo encontrarse em tal status de emprego, modelo Probit para o período de 2010. Os determinantes que apresentaram o maior impacto no sentido de reduzir as chances de pertencerem ao setor informal ou estarem desempregados foram: escolaridade, idade, estado civil, posição no domicílio e raça, além de uma composição de vizinhança adotada no estudo. Visando realizar uma comparação entre os gêneros, estima também as equações separadas por gêneros. Dentre as diferenças encontradas, a principal foi que, embora o impacto da educação sobre a probabilidade estudada tenha sido o mais importante dentre os fatores analisados para ambos os gêneros, ele se mostrou mais marcante entre as mulheres.

Palavras-chave: Mercado de trabalho; Informalidade; Desemprego; RMR.

Classificação JEL: J40; C01. 


\title{
DETERMINANTS OF INFORMALITY AND UNEMPLOYMENT IN THE LABOR MARKET IN THE METROPOLITAN REGION OF RECIFE IN THE PERIOD OF 2010
}

\begin{abstract}
In view of the importance of better understanding the labor market, the present study evaluates the behavior of the determinants of informality and unemployment through an econometric model that estimates the chances of an individual being in such employment status, the Probit model for the period of employment, 2010. The determinants that had the greatest impact in order to reduce the chances of belonging to the informal sector or being unemployed were: education, age, marital status, position at home and race, in addition to a neighborhood composition adopted in the study. In order to make a comparison between the genders, it also estimates the equations separated by genders. Among the differences found, the main one was that, although the impact of education on the probability studied was the most important among the factors analyzed for both genders, it was more striking among women.
\end{abstract}

Keywords: Labor market; Informality; Unemployment; RMR.

JEL classification: J40; C01. 


\section{INTRODUÇÃO}

A grande instabilidade da economia brasileira nos anos 1980 e 1990 agravou os problemas estruturais do mercado de trabalho. Destacam-se a precarização das formas de contratação, o crescimento do desemprego e a reversão da tendência ao aumento da formalização do trabalho assalariado, verificados no período anterior. À redução do emprego formal correspondeu a geração de outras oportunidades de ocupação não assalariadas e de empregos assalariados não formalizados. Como resultado, o crescimento da produção em pequena escala e do emprego à margem da regulamentação institucional têm reafirmado (ou intensificado) o caráter excludente e desigual do desenvolvimento brasileiro, e vêm desafiando a compreensão das possibilidades da modernização econômica em curso, especialmente no que concerne à organização e regulação do trabalho (BRAGA, 2016).

Desse ponto de vista, a investigação da informalização da ocupação fornece preciosas informações acerca da reconfiguração das formas de inserção no mercado de trabalho e do processo de transformações estruturais em andamento na economia e na própria sociedade brasileira. E, sem dúvida, tal afirmação também é válida para a Região Metropolitanas de Recife (RMR) que constitui o universo de análise deste estudo.

Dentre alguns artigos para a RMR, tem-se o estudo de Corrêa e Lima (2015) que analisaram os determinantes da inserção dos jovens da Região Metropolitana de Recife (RMR) no mercado de trabalho. Para tanto, foi estimada uma equação utilizando o procedimento de Heckman com dados da PNAD de 2012. Os resultados mostraram que ser do sexo masculino e chefe da família, maior idade e a escolaridade afetam positivamente a decisão do jovem de entrar no mercado de trabalho. Silva Filho, Queiroz e Clementino (2016) analisaram a participação feminina no mercado de trabalho no Brasil metropolitano, incluindo a RMR. Os dados foram da Pesquisa Nacional por Amostra de Domicílios (PNAD) e foram analisados no recorte que compreende os anos de 2001 e 2008. As principais estatísticas evidenciaram a elevação da participação feminina no mercado de trabalho com maior incidência na População Economicamente Ativa (PEA) nos anos em análise. 
Com o objetivo de realizar uma discussão mais pormenorizada sobre o fenômeno de interesse em termos regionais, o presente trabalho analisou os determinantes da informalidade e desemprego para a Região Metropolitana do Recife (RMR), avaliando o comportamento no mercado de trabalho nas chances do indivíduo estar desempregado ou no setor informal.

Além disso, procura-se analisar o comportamento da informalidade e do desemprego considerando cada gênero, visto que principalmente o desemprego não atinge homens e mulheres de forma homogênea. Para Hoffmann e Leone (2004), os processos de urbanização e industrialização, nas décadas de 1970 e 1980, foram acompanhados pela maior inserção das mulheres no mercado de trabalho. Nesse período, o desemprego não decorria da falta de absorção da mão de obra. Na década de 1990, porém, com o crescimento dos postos de trabalho em ritmo menor que o da PEA feminina, houve um aumento no desemprego entre mulheres. Conforme Lavinas, Amaral e Barros (2000), a taxa de desemprego feminina superou a masculina no final dessa década. Para Picchetti e Chahad (2003), o desemprego é maior entre as mulheres do que entre os homens devido ao preconceito do setor empresarial quanto à capacidade da força de trabalho feminina.

Para tanto, foram utilizados os dados do Censo $2010^{2}$ pelo Instituto Brasileiro de Geografia e Estatística (IBGE) em nível de área de ponderação. Especificamente, procurou-se identificar a existência ou não de papel decisivo na educação para os mercados de trabalho considerados, assim como comparar os resultados encontrados entre os sexos para a RMR.

O trabalho está dividido em mais três seções além desta introdução. A segunda seção apresenta a metodologia considerada nessa pesquisa. Na terceira seção, são apresentados os resultados e discussão, que, de modo geral, tratam da probabilidade do individuo estar na informalidade ou desempregado no mercado de trabalho da RMR. Por fim, a quarta e última seção exibe as considerações finais. 


\section{METODOLOGIA}

\section{1 Área de Estudo e Fonte dos Dados}

A área de estudo é a Região Metropolitana do Recife (RMR), que está localizada na Região Nordeste do Brasil, englobando 14 municípios $^{3}$ e tem uma população de aproximadamente 3.688.000 habitantes, conforme o censo demográfico de 2010, sendo a mais populosa do Nordeste e a quinta mais populosa do País (IBGE, 2011). A unidade de análise é o individuo dentro da Área Espacial de Ponderação ${ }^{4}$ (AEP), que é definida como uma área composta por um conjunto de zonas mutuamente exclusiva, projetado para dar a robustez estatística necessária à estratégia de amostragem do Censo de População (ver Figura 1).

Figura 1 - Áreas de Ponderação da região metropolitana do Recife.

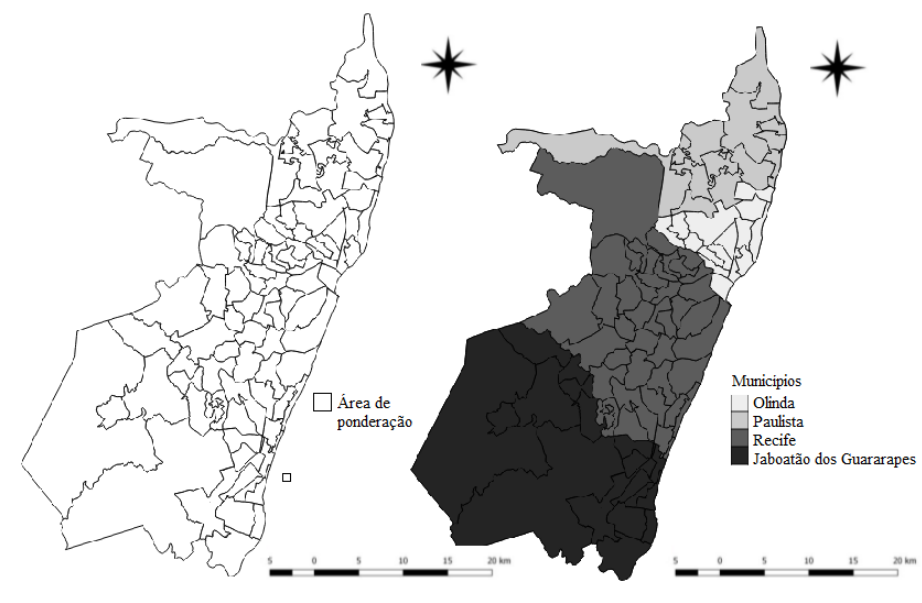

Fonte: Elaboração própria com base nos dados do Censo 2010.

Objetos deste trabalho, os municípios ${ }^{5}$ de Paulista, Olinda, Recife e Jaboatão dos Guararapes, representados na Figura 1, estão localizados no litoral do estado e fazem fronteira entre si. São os municípios mais populosos, juntos concentram 2.899.361 habitantes numa área de $611,57 \mathrm{~km}^{2}$ (densidade populacional de 4.740,85 hab $/ \mathrm{km}^{2}$ ). Juntos somam ainda um PIB per capita anual (a preços de 2012) de $\mathrm{R} \$ 557.041,54$ e têm rendimento domiciliar per capita anual de $\mathrm{R} \$ 2.423,29$. Embora representem apenas $22 \%$ da área total da RMR, os quatro municípios concentram $77 \%$ da população e $70 \%$ da renda da região (IBGE). Também fazem parte da RMR os municípios de Abreu e Lima, Araçoiaba, Cabo de Santo Agostinho, Camaragibe, Igarassu, Ilha de Itamaracá, Ipojuca, Itapissuma, 
Moreno e São Lourenço da Mata. Apenas os municípios de Camaragibe e São Lourenço da Mata fazem fronteira com Recife e não foram considerados no presente estudo.

Os microdados para as características individuais são obtidos no Censo $^{6}$ de População de 2010 (Instituto Brasileiro de Geografia e Estatística - IBGE, 2010). O IBGE realiza, a cada dez anos, um Censo Demográfico, com desagregação regional ao nível municipal (ou ao nível da área censitária dos municípios maiores). O Censo Demográfico coleta informações sobre as principais características dos indivíduos e famílias, fornecendo detalhes sobre as condições de vida da população em cada município e servindo como um instrumento de política muito importante em um país. Um questionário mais curto aplica-se a toda a população ao nível do setor censitário, enquanto as características individuais específicas são investigadas em um conjunto mais longo de questões que são dadas a uma amostra e são representativas ao nível das áreas ponderadas (conglomerados de setores censitários com pelo menos 400 domicílios). Para o presente trabalho foi utilizado áreas de ponderação como definição de vizinhança, uma vez que, microdados ao nível individual também estão disponíveis para esta amostra.

\subsection{Modelo Econométrico}

O modelo econométrico a ser apresentado pretende analisar os principais determinantes para a informalidade e desemprego no mercado de trabalho. Para tanto, investigou-se de que forma e com qual intensidade as características pessoais e domiciliares atuavam sobre a probabilidade de se encontrarem no setor informal e desempregado. Como nessa situação a variável dependente é binária, é necessário utilizar um modelo que seja capaz de tratar de tal limitação. Neste estudo, optou-se pelo modelo Probit, que estima a probabilidade com base na distribuição normal-padrão acumulada.

As estimativas são realizadas para indivíduos que residem em uma área específica, a fim de capturar o efeito de cada variável dentro de uma estrutura. O modelo Probit pode ser derivado, conforme apresentado em Greene (2003) e Wooldridge (2002), a partir de um modelo de variável latente. No presente trabalho, a variável dependente assume valor 1 caso o individuo pertença ao setor informal ou esteja desempregado. 
Seja Y* uma variável não-observada, ou latente, que representa o status do emprego (probabilidade de estar na informalidade ou desempregado, sendo determinada por:

$$
\operatorname{Prob}\left[\gamma_{i}=1\right]=\int_{-\infty}^{\beta} \Phi(t) d_{i}=\Phi\left(\beta^{\prime} x\right)
$$

onde a função $\Phi($.$) é a notação usual para a distribuição normal padrão cumulativa e \beta$ é o vetor de parâmetros das variáveis explicativas consideradas. Posteriormente, serão estimados os efeitos marginais, que serão considerados nas análises a seguir:

$$
\frac{\partial E[y \mid x]}{\partial x}=\Phi\left(x^{\prime} \beta\right) \beta
$$

Os efeitos marginais representam, por exemplo, o quanto uma mudança na variável educação pode afetar a probabilidade de um indivíduo estar desempregado. Os efeitos marginais (a variação da probabilidade de o evento ocorrer quando uma variável independente é modificada) são calculados por meio dos coeficientes estimados $\beta^{\prime}$ s.

A equação geral do Modelo em função das variáveis explicativas adotadas é representada por:

$$
\Phi\left(\beta^{\prime} x\right)=\Phi\left(\beta_{0}+\beta_{1} X_{i}+\beta_{2} H_{j}+\beta_{3} V_{j}+\mu\right)
$$

Em que $X_{i}$ denota o vetor de características pessoais; $H_{j}$ representa o vetor de características domésticas; $V_{j}$ é um vetor de características de vizinhança (composição social); e $\beta$ são vetores de parâmetros que serão estimados; e $\mu$, o termo de erro aleatório com distribuição normal-padrão. As variáveis explicativas utilizadas na equação podem ser observadas no Quadro 1, a seguir. Para determinar o status de cada trabalhador, utilizamos variáveis sobre status ocupacional e tipo de emprego (no trabalho principal). 
Quadro 1 - Descrição das variáveis dos modelos econométricos.

\begin{tabular}{|c|c|}
\hline \multicolumn{2}{|r|}{ Amostra } \\
\hline VARIÁVEIS & DESCRIÇÃO \\
\hline Informal & Se a pessoa estar na informalidade. \\
\hline Desemprego & Se a pessoa estar desempregada. \\
\hline Masculino & $\begin{array}{l}\text { Variável dummy se for do sexo } \\
\text { masculino. }\end{array}$ \\
\hline Idade & Em anos. \\
\hline Idade $^{2}$ & Proxy para experiência. \\
\hline $\begin{array}{l}\text { Educação } \\
\text { (dummies) } \\
\text { i) Baixo } \\
\text { ii) } \\
\text { Intermediário } \\
\text { iii) Alto } \\
\text { iv) Bem } \\
\text { elevado }\end{array}$ & $\begin{array}{l}\text { i) Sem ensino fundamental } \\
\text { completo; } \\
\text { ii) Com ensino fundamental } \\
\text { completo, mas sem médio completo; } \\
\text { iii) Com ensino médio completo, } \\
\text { mas sem superior; } \\
\text { iv) Com superior completo ou mais. }\end{array}$ \\
\hline Estado Civil & $\begin{array}{l}\text { Variável dummy se o individuo for } \\
\text { casado }\end{array}$ \\
\hline $\begin{array}{l}\text { Posição no } \\
\text { domicílio } \\
\text { (dummy) }\end{array}$ & $\begin{array}{l}\text { Chefe } \\
\text { Cônjuge } \\
\text { Filho }\end{array}$ \\
\hline $\begin{array}{l}\text { Raça } \\
\text { (dummies) }\end{array}$ & $\begin{array}{l}\text { Branco } \\
\text { Pardo } \\
\text { Negro } \\
\text { Amarelo }\end{array}$ \\
\hline$T_{\text {f familia }}$ & Tamanho da família \\
\hline Localidade & $\begin{array}{l}\text { Variável dummy se o domicilio } \\
\text { pertence a área urbana. }\end{array}$ \\
\hline Vizinhança & $\begin{array}{l}\text { Muito Carente } \\
\text { Carente } \\
\text { Media } \\
\text { Desenvolvida } \\
\text { Muito Desenvolvida }\end{array}$ \\
\hline $\begin{array}{l}\text { Acesso à } \\
\text { infraestrutura } \\
\text { (dummies) }\end{array}$ & $\begin{array}{l}\text { Coleta de lixo } \\
\text { Saneamento básico }\end{array}$ \\
\hline
\end{tabular}

Fonte: Elaboração própria. 
No que diz respeito aos conceitos de informalidade, as diversas definições podem levar a resultados substancialmente diferentes, dificultando uma comparação entre estudos. Uma classificação tradicional na literatura brasileira enquadra como trabalhadores informais, àqueles que não possuem carteira de trabalho assinada, tal como o trabalho de Fernandes (1996), Carneiro e Henley (2001), Pianto e Pianto (2002) e Menezes-Filho, Mendes e Almeida (2004). Estudos recentes, como os de Dalberto (2014), Cirino e Dalberto (2014) estabelecem como conceito de trabalhador informal aquele que não contribui para o Instituto Nacional do Seguro Social. Outros trabalhos como de Machado, Oliveira e Antigo (2008) e Duarte et al., (2018) combina a definição de regulação do trabalho, ou seja, carteira assinada como característica de formalidade e sem carteira assinada como informalidade, e a definição de subordinação. Dessa forma, o trabalhador informal é o que se auto declara conta própria, exceto as ocupações de profissionais liberais, e os empregados sem carteira assinada.

Apesar de existirem diversas definições para a informalidade, no trabalho foi considerada a definição mais simples possível, o trabalhador apenas sem carteira assinada para representar a informalidade e o trabalhador que está na População Economicamente Ativa (PEA), mas se encontra desocupado para representar o individuo desempregado.

Mais especificamente, as variáveis pessoais absorvem as diferentes preferências com relação ao espaço de acordo com certas características. Por exemplo, quanto à dummy para gênero, regularmente, observa-se nos trabalhos empíricos que as mulheres tendem a se localizar mais próximos aos locais de trabalho, o que é comumente atribuído à noção de maior responsabilidade para com as tarefas com lar atribuído às mulheres o que podem aumentar a informalidade (LEE \& MACDONALD, 2003; CRAINE, 2007). De forma similar, a condição de casado pode atribuir um caráter mais permanente à localização dos domicílios e, assim, menor vinculação imediata ao local de trabalho. As variáveis quanto à idade e de escolaridade traduzem possíveis influências da disposição à locomoção e preferências quanto à vizinhança por parte dos indivíduos. 
Discutindo as hipóteses acerca do relacionamento entre as variáveis explicativas consideradas e a probabilidade do individuo estar na informalidade ou desempregado $\left(Y_{i}\right)$, espera-se, a priori, um relacionamento negativo quanto à escolaridade, já que quanto mais anos de estudo, melhores as oportunidades de emprego e as chances de acesso a rendas elevadas.

Com relação à idade e idade ${ }^{2}$, utilizada como proxy da experiência, acredita-se em uma relação negativa também. Tal relacionamento indica que as oportunidades de trabalho e os salários aumentam à medida que a pessoa adquire mais experiência até certo ponto. Em relação à condição no domicilio, é esperado que os chefes apresentem menor probabilidade de serem informais ou desempregados do que os outros grupos, por serem aqueles geralmente os responsáveis por grande parte do sustento de seus membros familiares.

Passando para os coeficientes das variáveis de infraestrutura, acredita-se que eles sejam negativos, visto que ter melhor acesso ao saneamento e coleta de lixo, podem indicar menores as chances de informalidade e desemprego. O mesmo raciocínio vale para as variáveis que compõem a vizinhança.

\subsection{Definição das Características da vizinhança}

Neste estudo foi utilizado um indicador para explicar as características da vizinhança que podem potencialmente influenciar as probabilidades individuais de desemprego e informalidade no mercado de trabalho. Em relação às variáveis que caracterizam a composição social da vizinhança, os pesquisadores geralmente recorrem a uma ou várias medidas quantitativas das características agregadas dos residentes. Contudo, embora seja provável que os resultados individuais sejam determinados por uma ampla variedade de características de vizinhança (emprego, educação, composição racial etc.), considerar todas essas características juntas em uma única regressão podem causar problemas de colinearidade, já que muitos indicadores de composição de vizinhança são altamente inter-relacionados (O’REGAN \& QUIGLEY, 1996; JOHNSTON et al., 2004). Para contornar esse problema, foi utilizado métodos de análise fatorial padrão para resumir essas múltiplas características em diferentes tipos de vizinhança nas AEP da RMR. 
Tabela 1 -Variáveis usadas na análise de componentes principais e seus fatores.

\begin{tabular}{lc}
\hline & \multicolumn{1}{l}{ Fator } \\
\hline Autovalores & $\mathbf{1}$ \\
\hline Porcentagem de variação explicada & 59,726 \\
\hline Variáveis & 59,08 \\
\hline \% de Mulheres chefes & $-0,250$ \\
\hline$\%$ de pessoas com baixo nível educacional & 0,443 \\
\hline$\%$ de pessoas com ensino intermediário & $-0,305$ \\
\hline$\%$ de pessoas com alto nível educacional & $-0,412$ \\
\hline \% de pessoas desocupadas & $-0,319$ \\
\hline \% de domicílios sem acesso à rede de & 0,328 \\
\hline saneamento & \\
\hline \% de pessoas sem acesso a coleta de lixo & 0,288 \\
\hline \% de pessoas com menos de 15 anos & $-0,430$ \\
\hline
\end{tabular}

Notas: Apenas fatores com valores próprios superiores ou iguais a 1 foram selecionados.

O método de análise fatorial foi usado para identificar áreas socialmente homogêneas dentro da RMR, que serão subsequentemente usadas nas análises de regressão. Primeiro foi executado uma análise de componentes principais que define um número limitado de fatores não correlacionados resumindo um conjunto de informações da vizinhança como percentual de mulheres chefes, nível educacional, percentual de pessoas desocupadas, percentual de domicílios sem acesso à rede de saneamento e coleta de lixo (Tabela 1).

Em seguida, as áreas são agrupadas de acordo com suas coordenadas nos eixos fatoriais, usando uma classificação hierárquica ascendente (ver método Ward, que minimiza a variância intragrupo). Segundo Hair et al., (2005), o método de Ward consiste em um procedimento de agrupamento hierárquico no qual a medida de similaridade usada para juntar agrupamentos é calculada como a soma de quadrados entre os dois agrupamentos feita sobre todas as variáveis. Esse método tende a resultar em agrupamentos de tamanhos aproximadamente iguais devido a sua minimização de variação interna. Em cada estágio, combinam-se os dois agrupamentos que apresentarem menor aumento na soma global de quadrados dentro dos agrupamentos. 
Foram obtidos cinco tipos de vizinhança (Tabela 2). Este foi o número ideal de clusters de acordo com vários critérios, incluindo o critério de agrupamento cúbico.

Tabela 2 - Média das características dos tipos de vizinhança.

\begin{tabular}{|c|c|c|c|c|c|c|}
\hline & $\begin{array}{l}\text { Muito } \\
\text { Carente }\end{array}$ & Carente & Média & Desenvolvido & $\begin{array}{l}\text { Muito } \\
\text { Desenvolvido }\end{array}$ & Total \\
\hline$\%$ de Mulheres chefes & 12,8 & 13,5 & 14,9 & 14,1 & 15,3 & 13,8 \\
\hline $\begin{array}{l}\% \text { de pessoas com baixo nível } \\
\text { educacional }\end{array}$ & 77,9 & 70,3 & 50,9 & 59,8 & 30,8 & 65,0 \\
\hline $\begin{array}{l}\% \text { de pessoas com ensino } \\
\text { intermediário }\end{array}$ & 19,5 & 24,9 & 31,7 & 29,5 & 28,3 & 25,7 \\
\hline $\begin{array}{l}\% \text { de pessoas com alto nível } \\
\text { educacional }\end{array}$ & 1,9 & 4,0 & 16,8 & 10,0 & 40,4 & 8,6 \\
\hline$\%$ de pessoas desocupadas & 7,1 & 6,6 & 5,3 & 6,2 & 3,5 & 6,3 \\
\hline $\begin{array}{l}\% \text { de domicílios sem acesso à } \\
\text { rede de saneamento }\end{array}$ & 77,6 & 63,5 & 31,2 & 45,6 & 10,0 & 55,6 \\
\hline $\begin{array}{l}\% \text { de pessoas sem acesso a } \\
\text { coleta de lixo }\end{array}$ & 7,6 & 5,2 & 1,2 & 1,6 & 0,1 & 4,2 \\
\hline $\begin{array}{l}\% \text { de pessoas com menos de } 15 \\
\text { anos }\end{array}$ & 25,8 & 23,9 & 18,3 & 20,6 & 13,8 & 22,3 \\
\hline
\end{tabular}

Nota: Os cinco tipos de vizinhança foram definidos por uma classificação hierárquica ascendente com o Critério de Ward.

O primeiro tipo de vizinhança compreende áreas muito carentes com percentual alto da população com baixo nível educacional, famílias chefiadas por mulheres, sem acesso a saneamento básico e com maior quantidade de pessoas com menos de 15 anos. Estas áreas também possuem os níveis de desemprego mais alto de toda a aglomeração. Eles estão cercados por um grupo de áreas carentes que apresentam características semelhantes, mas com uma proporção menor de famílias sem acesso a saneamento e uma situação menos grave em termos de educação e desocupação. O grupo 3 (Média) têm um nível socioeconômico mais baixo, em particular uma super-representação de trabalhadores e indivíduos com menor escolaridade. No entanto, a taxa de desocupação está próxima da média da região. Os dois grupos restantes são caracterizados em média por níveis mais altos de educação e percentuais baixos de domicílios sem acesso a saneamento básico e com pessoas com menos de 15 anos. Eles dois últimos grupos apresentam os melhores indicadores entre todas as vizinhanças construídas na RMR. 


\subsection{Limitação do estudo}

Existem algumas limitações com os dados utilizados neste estudo. Em primeiro lugar, os dados mais atuais para o Censo é para o ano de 2010, visto que não foram disponibilizados oficialmente os dados do Censo 2020. Em segundo lugar, o status de informalidade é derivado dos dados do recenseamento e, portanto, depende de pessoas que relatem com sinceridade seu status. Como algumas pessoas podem querer disfarçar que estão trabalhando informalmente, os dados do estudo podem estar superestimando o número de indivíduos formalmente empregados. No entanto, dado o tamanho da amostra relativamente grande, os dados permitem explorar a relação entre informalidade e seus determinantes. Por fim, em terceiro lugar, as variáveis geográficas são geradas em nível agregado por área de ponderação em que os indivíduos residem. Consequentemente, isso pode não refletir completamente o impacto inicial dos indivíduos. No entanto, esse tipo de análise agregada permite explorar os padrões a nível regional.

\section{RESULTADOS}

As médias e os desvios padrão das variáveis incluídas no modelo para o ano de 2010, levando em conta os dados do Censo, são apresentados na Tabela 3, a seguir. No caso das variáveis dummies, a média é a proporção de casos em que a variável assume o valor 1.

Em relação aos anos de estudo, as estatísticas confirmaram que os indivíduos apresentam, em média, nível educacional mais baixo, com maiores taxas para indivíduos sem ensino fundamental completo. Quanto à posição no domicílio, verificou-se, que os chefes e filhos são a maioria, representando, em geral, $30 \%$ e $34 \%$ do percentual da amostra, respectivamente (Tabela 3 ). Os dados mostrados ainda apresentaram a existência de maior proporção de pessoas que se declaram brancas e pardas na RMR. 
Tabela 3 - Estatística Descritiva das variáveis da equação no mercado de trabalho, 2010.

\begin{tabular}{|c|c|c|c|c|}
\hline Variáveis & Média & $\begin{array}{l}\text { Desvio } \\
\text { Padrão }\end{array}$ & Mínimo & Máximo \\
\hline Informal & 0,080 & 0,269 & 0 & 1 \\
\hline Desemprego & 0,062 & 0,242 & 0 & 1 \\
\hline Masculino & 0,468 & 0,499 & 0 & 1 \\
\hline Idade & 32,42 & 20,25 & 0 & 109 \\
\hline Idade $^{2}$ & 1461,4 & 1570,6 & 0 & 11881 \\
\hline \multicolumn{5}{|l|}{ Escolaridade } \\
\hline Baixo & 0,504 & 0,499 & 0 & 1 \\
\hline Intermediário & 0,149 & 0,356 & 0 & 1 \\
\hline Alto & 0,258 & 0,438 & 0 & 1 \\
\hline Bem Elevado & 0,086 & 0,281 & 0 & 1 \\
\hline \multicolumn{5}{|l|}{ Estado Civil } \\
\hline Casado & 0,263 & 0,440 & 0 & 1 \\
\hline \multicolumn{5}{|l|}{ Posição no domicílio } \\
\hline Chefe & 0,300 & 0,458 & 0 & 1 \\
\hline Cônjuge & 0,181 & 0,385 & 0 & 1 \\
\hline Filho & 0,344 & 0,475 & 0 & 1 \\
\hline Outro & 0,174 & 0,379 & & \\
\hline \multicolumn{5}{|l|}{ Raça } \\
\hline Branca & 0,385 & 0,486 & 0 & 1 \\
\hline Negro & 0,087 & 0,283 & 0 & 1 \\
\hline Pardo & 0,515 & 0,499 & 0 & 1 \\
\hline Amarelo & 0,011 & 0,106 & 0 & 1 \\
\hline T_familia & 4,146 & 1,948 & 1 & 22 \\
\hline \multicolumn{5}{|l|}{ Localidade } \\
\hline Urbano & 0,992 & 0,087 & 0 & 1 \\
\hline \multicolumn{5}{|l|}{ Vizinhança } \\
\hline Muito Carente & 0,233 & 0,423 & 0 & 1 \\
\hline Carente & 0,381 & 0,485 & 0 & 1 \\
\hline Media & 0,162 & 0,368 & 0 & 1 \\
\hline Desenvolvida & 0,167 & 0,373 & 0 & 1 \\
\hline Muito Desenvolvida & 0,054 & 0,227 & 0 & 1 \\
\hline \multicolumn{5}{|l|}{ Infraestrutura } \\
\hline Coleta & 0,899 & 0,300 & 0 & 1 \\
\hline Saneamento & 0,434 & 0,495 & 0 & 1 \\
\hline
\end{tabular}

Fonte: Elaboração própria com base nos dados da pesquisa. 
A estimação das equações foram realizadas, separadamente, para homens e mulheres, sendo os resultados gerais para a RMR apresentados na Tabela 4 e aqueles referentes a gêneros apresentados na Tabela 5. No que tange à significância, observou-se que a grande maioria das variáveis foi significativa a 1\%, ressaltando a importância de tais regressores para a explicação da probabilidade do individuo ser informal ou estar desempregado no mercado de trabalho.

Observando-se as estimativas, nota-se que, embora o coeficiente de determinação da regressão $\left(\mathrm{R}^{2}\right)$ não seja muito elevado, a estimativa para os coeficientes apresentam valores elevados e com sinal esperado. A estatística LR chi $^{2}$ é estatisticamente significativa e assume um valor que facilmente excede 10.

Com relação aos resultados, todas as variáveis incorporadas foram importantes para a explicação da situação dos indivíduos no mercado de trabalho. No que se refere ao mercado de trabalho para a RMR, no geral, a informalidade e desemprego foram influenciadas pela escolaridade, idade, estado civil, raça, vizinhança e infraestrutura (Tabela 4). 
Tabela 4 - Resultado das equações para informalidade na RMR, 2010.

\begin{tabular}{|c|c|c|c|c|}
\hline \multirow[b]{2}{*}{ Variáveis } & \multicolumn{2}{|c|}{ INFORMALIDADE } & \multicolumn{2}{|c|}{ DESEMPREGO } \\
\hline & E.M. & D.P & E.M. & D.P \\
\hline Masculino & $-0,099 * * *$ & 0,011 & $-0,064 * * *$ & 0,002 \\
\hline Idade & $-0,010^{* * *}$ & 0,002 & $-0,009^{* * *}$ & 0,001 \\
\hline Idade $^{2}$ & $0,001 * * *$ & 0,000 & $0,001 * * *$ & 0,000 \\
\hline Baixo & Ref. & Ref. & Ref. & Ref. \\
\hline Intermediário & $-0,058 * * *$ & 0,016 & $0,006^{*}$ & 0,003 \\
\hline Alto & $-0,131 * * *$ & 0,014 & $-0,023 * * *$ & 0,002 \\
\hline Bem Elevado & $-0,158^{* * *}$ & 0,021 & $-0,077 * * *$ & 0,002 \\
\hline Casado & $-0,056^{* * *}$ & 0,013 & $-0,031 * * *$ & 0,002 \\
\hline Chefe & Ref. & Ref. & Ref. & Ref. \\
\hline Cônjuge & $-0,001$ & 0,014 & $0,028 * * *$ & 0,003 \\
\hline Filho & 0,004 & 0,016 & $0,072 * * *$ & 0,003 \\
\hline Outro & $0,002 * * *$ & 0,005 & $0,035^{* * *}$ & 0,004 \\
\hline Branco & Ref. & Ref. & Ref. & Ref. \\
\hline Negro & $0,015^{* * *}$ & 0,019 & $0,077^{*}$ & 0,004 \\
\hline Pardo & $0,008 * * *$ & 0,012 & $0,011 * * *$ & 0,009 \\
\hline Amarelo & 0,010 & 0,050 & $-0,013$ & 0,001 \\
\hline T_Familia & $0,004 * * *$ & 0,003 & $0,003^{* * *}$ & 0,009 \\
\hline Urbano & $-0,013$ & 0,065 & 0,001 & 0,001 \\
\hline Muito Carente & Ref. & Ref. & Ref. & Ref. \\
\hline Carente & 0,001 & 0,015 & $-0,009^{* * *}$ & 0,002 \\
\hline Media & 0,002 & 0,019 & $-0,025^{* * *}$ & 0,003 \\
\hline Desenvolvida & $0,016 * * *$ & 0,018 & $-0,015^{* * *}$ & 0,003 \\
\hline Muito Desenvolvida & $-0,009$ & 0,029 & $-0,041 * * *$ & 0,004 \\
\hline Coleta & $-0,019 * * *$ & 0,019 & $-0,007 *$ & 0,003 \\
\hline Saneamento & $-0,013 * * *$ & 0,012 & $-0,003$ & 0,002 \\
\hline Constante & - & - & - & - \\
\hline Observações & 70.626 & & Observações & 81.454 \\
\hline Pseudo R 2 & 0.0772 & & Pseudo R 2 & 0,1044 \\
\hline Prob $>\mathrm{chi}^{2}$ & 0,0000 & & Prob $>\mathrm{chi}^{2}$ & 0,0000 \\
\hline LR chi ${ }^{2}$ & 5347,50 & & LR chi $^{2}$ & 6664,86 \\
\hline
\end{tabular}

Fonte: Resultados da Pesquisa. Nota: Desvio-padrão entre parênteses. ${ }^{* * *} p<0.01, * * p<0.05$, ${ }^{*}$ p $<0.1$. E.M.: efeito marginal. 
Quanto aos coeficientes estimados, é importante ressaltar que, embora a magnitude deles não apresente significado econômico importante na maioria dos casos, os seus sinais indicam os sentidos dos efeitos marginais, isto é, as direções das mudanças de probabilidades, dadas as alterações nas variáveis explicativas (WOOLDRIDGE, 2002). Nesse sentido, observou-se que os sinais e, consequentemente os efeitos marginais comportaram-se de acordo com o esperado. Sobre os efeitos marginais, é importante destacar que a sua interpretação em modelos de variável dependente binária, é a seguinte: i) para variáveis contínuas, representa o impacto médio em termos de pontos percentuais na probabilidade de um individuo estar na informalidade ou desempregado para aumento unitário da variável explicativa contínua considerada; e ii) para as variáveis qualitativas, determina a mudança média na probabilidade de um indivíduo estar no setor informal ou desempregado, em pontos percentuais, devido à presença da característica indicada pela dummy considerada.

Iniciando a discussão dos determinantes do status do individuo no mercado de trabalho, verificou-se a ocorrência de sinal negativo para a dummy de sexo, refletindo o efeito menor para trabalhadores do sexo masculino em estarem na condição de trabalhador informal ou desempregado (Tabela 5). Infelizmente, a divisão tradicional das atividades familiares e a consequente maior experiência da mulher em atividades domésticas direcionam essa força de trabalho secundária para o emprego de serviços domésticos (BALSADI, 2000). Outra explicação é que, na maioria dos casos, as mulheres não são chefes de famílias, portanto, buscam empregos parciais, sazonais ou temporários menos remunerados do que empregos em tempo integral. Além disso, o duplo papel de trabalhadora, ou seja, ser dona de casa/mãe restringe os deslocamentos para buscarem empregos no setor formal.

Em relação à idade, os resultados mostraram que quanto mais experiência para o trabalhador, menores as chances de pertencerem na informalidade ou desempregados no mercado de trabalho da RMR. Mesmo resultado obtido quando considera o individuo casado. Isso pode ser explicado pelo fato de que os melhores salários pagos são os trabalhos formais, dessa forma, os indivíduos estão mais dispostos a buscarem melhores empregos para obter condições para sustento da família. Então ser 
casado, diminui as chances de pertencer ao setor informal e estarem desempregados em 0,056 e 0,031 pontos percentuais, respectivamente.

Com relação à educação, conforme esperado, todos os coeficientes apresentaram sinais negativos, já que o grupo-base adotado foram os indivíduos com menor grau de instrução. Dessa forma, em relação a esses últimos, as pessoas pertencentes às demais categorias apresentaram maiores chances de pertencerem ao setor informal ou estarem no desemprego, ou seja, esta probabilidade diminui conforme o aumento da escolaridade. Percebe-se que a magnitude dos efeitos marginais também aumentem a medida que aumenta o grau de instrução.

Passando para a variável de posição no domicílio, verificou-se, no mercado, que não houve diferença estatística entre os trabalhadores chefes e para os outros no domicílio nos resultados para a informalidade. Contudo, analisando o desemprego, percebe-se que o chefe de domicilio tem menor probabilidade de estar desempregado em comparação com as demais posições. Na Tabela 4, o fato de o individuo chefe ser a pessoa de referencia faz que a referida probabilidade seja de 0,$028 ; 0,072$ e 0,035 pontos percentuais menores do que se ele fosse cônjuge, filho ou outro, respectivamente.

Quanto ao impacto da raça sobre a probabilidade dos indivíduos estarem na informalidade ou desempregados, verificou-se que o fato de serem negros ou pardos faz com que a probabilidade seja superior em comparação com o grupo de brancos. Tal resultado para a informalidade pode estar refletindo a maior necessidade de trabalhar daquelas raças em virtude de, geralmente, elas se situarem em classes sociais mais baixas do que as brancas (Cirino \& De Lima, 2011), fazendo com que trabalhar no setor informal seja uma forma de não permanecer desempregada. Além do mais, sabe-se que existe a discriminação no mercado de trabalho, onde trabalhadores negros sofrem com isso constantemente.

O tamanho da família também influencia na probabilidade de forma positiva, ou seja, quanto maior o número de pessoas no domicilio, maiores as chances do indivíduo estar no setor informal ou desempregado. Isso reflete a situação das famílias mais carentes no Brasil, onde, geralmente moram no mesmo domicílio três gerações, filhos, pais e avós. Em relação as variável que mede a localidade do domicilio, esta não demonstrou diferença estatística para as variáveis dependentes estudadas. 
Os resultados confirmam que o tipo da vizinhança têm um efeito negativo no emprego. Moradores de áreas muito carentes têm menor probabilidade de encontrar um emprego. Os resultados do presente estudo corroboram com os resultados obtidos em outros trabalhos para Economia Urbana. Gobillon e Selod (2006) concluem que a segregação residencial impede que trabalhadores desempregados encontrem um emprego, enquanto Dujardin et al., (2008) confirmam que residir em um bairro pobre aumenta a probabilidade de estar desempregado. Já para a informalidade, a maioria dos coeficientes não foram significativos.

Por fim, quanto as variáveis que representam a infraestrutura, os domicílios com melhor acesso a coleta de lixo, diminui a chance estarem na informalidade ou desempregados, enquanto que, a falta de saneamento básico, diminui essa probabilidade apenar para o individuo do setor informal (Tabela 4).

A relação entre os determinantes da informalidade e desemprego mostram padrões mais distintos quando permite a heterogeneidade entre os gêneros. Nas Tabelas 5 e 6 têm-se efeitos heterogêneos para homens e mulheres. Observando todos os efeitos marginais, percebe-se que os sinais parecem corroborar com a literatura para a escolaridade, por exemplo, trabalhadores mais escolarizados tem menor probabilidade de trabalhar no setor informal. Comparados aos resultados gerais, não houve muita diferença entre os efeitos e sinais esperados. Os resultados para idade, escolaridade, casado e infraestrutura permanecem no mesmo padrão. Contudo, pode-se observar que algumas variáveis mudam seu efeito quando comparadas entre os sexos.

O tamanho da informalidade e desemprego econômico é uma resposta à incapacidade da economia formal para acomodar todos os trabalhadores, então a estrutura econômica formal determinará quais indivíduos se dedicam a atividades econômicas informais, ao desemprego e onde residem (não onde eles decidem viver). Observando a composição da vizinhança, percebe-se que comparados com o grupo base "Carente", morar em uma vizinhança com melhores indicadores diminuem a probabilidade de pertencerem ao setor informal ou estarem desempregados para ambos os sexos. Ressalta-se que, apenas para os homens duas dessas variáveis de vizinhança foram positivas comparadas ao grupo de referência. Isso mostra que para o mercado de trabalho informal dos homens, segue um padrão um pouco distinto 
comparado às mulheres, devido que, em geral, cabe ao gênero masculino maior imposição na busca por emprego, sendo que na maioria das vezes não é possível o trabalho formal, tendo que recorrer à informalidade.

Tabela 5 - Resultado das equações para informalidade por gênero para a RMR, 2010.

\begin{tabular}{|c|c|c|c|c|}
\hline \multirow{2}{*}{ Variáveis } & \multicolumn{2}{|c|}{ Homens } & \multicolumn{2}{|c|}{ Mulheres } \\
\hline & E.M. & D.P & E.M. & D.P \\
\hline Idade & $-0,013 * * *$ & 0,001 & $-0,007 * * *$ & 0,001 \\
\hline Idade $^{2}$ & $0,001 * * *$ & 0,000 & $0,001 * * *$ & 0,000 \\
\hline Baixo & Ref. & Ref. & Ref. & Ref. \\
\hline Intermediário & $-0,042 * * *$ & 0,004 & $-0,085 * * *$ & 0,005 \\
\hline Alto & $-0,091 * * *$ & 0,003 & $-0,188 * * *$ & 0,005 \\
\hline Bem Elevado & $-0,103 * * *$ & 0,004 & $-0,225 * * *$ & 0,004 \\
\hline Casado & $-0,049 * * *$ & 0,004 & $-0,053 * * *$ & 0,005 \\
\hline Chefe & Ref. & Ref. & Ref. & Ref. \\
\hline Cônjuge & $0,031 * * *$ & 0,005 & $-0,039 * * *$ & 0,005 \\
\hline Filho & $0,035 * * *$ & 0,006 & $-0,039 * * *$ & 0,006 \\
\hline Outro & 0,029 & 0,007 & $-0,032 * * *$ & 0,007 \\
\hline Branco & Ref. & Ref. & Ref. & Ref. \\
\hline Negro & 0,002 & 0,006 & $0,034 * * *$ & 0,009 \\
\hline Pardo & $-0,003$ & 0,003 & $0,025 * * *$ & 0,005 \\
\hline Amarelo & $-0,014$ & 0,016 & $0,035^{*}$ & 0,021 \\
\hline $\mathrm{T}_{\text {_familia }}$ & $0,002 * *$ & 0,000 & $0,005 * *$ & 0,001 \\
\hline Urbano & $-0,019$ & 0,020 & $-0,010$ & 0,032 \\
\hline Muito Carente & Ref. & Ref. & Ref. & Ref. \\
\hline Carente & 0,005 & 0,004 & $-0,007$ & 0,006 \\
\hline Media & $0,012 * *$ & 0,006 & $-0,013^{*}$ & 0,008 \\
\hline Desenvolvida & $0,025 * * *$ & 0,006 & $-0,001$ & 0,007 \\
\hline Muito Desenvolvida & 0,006 & 0,010 & $-0,033 * * *$ & 0,011 \\
\hline Coleta & $-0,017 * * *$ & 0,006 & $-0,024 * * *$ & 0,008 \\
\hline Saneamento & $-0,004$ & 0,003 & $-0,026 * * *$ & 0,005 \\
\hline Constante & - & - & - & - \\
\hline Observações & 39.357 & & 31.269 & \\
\hline Pseudo $\mathrm{R}^{2}$ & 0,0588 & & 0,0894 & \\
\hline LR chi ${ }^{2}$ & 1984.92 & & 3060.23 & \\
\hline
\end{tabular}

Fonte: Resultados da Pesquisa. Nota: Desvio-padrão entre parênteses. ***p $<0.01, * * \mathrm{p}<0.05$, $*$ p $<0.1$. E.M.: efeito marginal.

Em termos de tamanho do efeito marginal da escolaridade, verificou-se que, de maneira geral, tal variável provocou grande impacto sobre a probabilidade de o individuo no mercado de trabalho, 
principalmente entre as mulheres. Em termos de diferenças entre os gêneros, verificou-se que os efeitos marginais das mulheres se apresentaram superiores aos dos homens, indicando que as mulheres são mais sensíveis. Tal resultado reforça o importante papel da escolaridade para a redução da informalidade feminina no mercado de trabalho (Tabela 5).

Tabela 6 - Resultado das equações para desemprego por gênero para a RMR, 2010.

\begin{tabular}{|c|c|c|c|c|}
\hline \multirow{2}{*}{ Variáveis } & \multicolumn{2}{|c|}{ Homens } & \multicolumn{2}{|c|}{ Mulheres } \\
\hline & E.M. & D.P & E.M. & D.P \\
\hline Idade & $-0,006 * * *$ & 0,001 & $-0,012 * * *$ & 0,001 \\
\hline Idade $^{2}$ & $0,001 * * *$ & 0,000 & $0,001 * * *$ & 0,000 \\
\hline Baixo & Ref. & Ref. & Ref. & Ref. \\
\hline Intermediário & $-0,001$ & 0,003 & $0,018 * * *$ & 0,005 \\
\hline Alto & $-0,023 * * *$ & 0,003 & $-0,023 * * *$ & 0,004 \\
\hline Bem Elevado & $-0,058 * * *$ & 0,003 & $-0,098 * * *$ & 0,004 \\
\hline Casado & $-0,029 * * *$ & 0,003 & $-0,026 * * *$ & 0,004 \\
\hline Chefe & Ref. & Ref. & Ref. & Ref. \\
\hline Cônjuge & $0,018 * * *$ & 0,004 & $0,018 * * *$ & 0,004 \\
\hline Filho & $0,104 * * *$ & 0,004 & $0,029 * * *$ & 0,005 \\
\hline Outro & $0,072 * * *$ & 0,006 & $-0,006$ & \\
\hline Branco & Ref. & Ref. & Ref. & Ref. \\
\hline Negro & 0,001 & 0,004 & $0,016^{* * *}$ & 0,007 \\
\hline Pardo & $0,005^{*}$ & 0,002 & $0,019 * * *$ & 0,004 \\
\hline Amarelo & $-0,032 * * *$ & 0,010 & 0,003 & 0,015 \\
\hline $\mathrm{T}$ _familia & 0,001 & 0,001 & $0,006^{* * *}$ & 0,001 \\
\hline Urbano & $-0,005$ & 0,014 & 0,001 & 0,023 \\
\hline Muito Carente & Ref. & Ref. & Ref. & Ref. \\
\hline Carente & $-0,005^{*}$ & 0,003 & $-0,013 * * *$ & 0,004 \\
\hline Media & $-0,016^{* *}$ & 0,004 & $-0,036 * * *$ & 0,005 \\
\hline Desenvolvida & $-0,011 * * *$ & 0,003 & $-0,022 * * *$ & 0,005 \\
\hline Muito Desenvolvida & $-0,031 * * *$ & 0,005 & $-0,050 * * *$ & 0,007 \\
\hline Coleta & $-0,003$ & 0,004 & $-0,011^{*}$ & 0,006 \\
\hline Saneamento & $-0,001$ & 0,002 & $-0,005$ & 0,004 \\
\hline Constante & - & - & - & - \\
\hline Observações & 43.886 & & Observações & 37.568 \\
\hline Pseudo $\mathrm{R}^{2}$ & 0,1054 & & Pseudo $\mathrm{R}^{2}$ & 3260,69 \\
\hline LR chi $^{2}$ & 3072,59 & & LR chi $^{2}$ & 0,0960 \\
\hline
\end{tabular}

Fonte: Resultados da Pesquisa. Nota: Desvio-padrão entre parênteses. ${ }^{* *}$ p $<0.01, * * p<0.05$, $*_{p}<0.1$. E.M.: efeito marginal. 


\section{CONSIDERAÇÕES FINAIS}

O presente trabalho analisou os determinantes para a informalidade e desemprego nos mercados de trabalho da Região Metropolitana do Recife (RMR) a partir dos dados do Censo 2010 por área de ponderação. Os resultados apresentados forneceram uma visão da probabilidade de pertencerem nesses status de emprego.

As variáveis escolaridades, idade, estado civil, posição no domicílio, raça, composição da vizinhança e acesso à coleta de lixo, apresentaram-se como determinantes significativos para a probabilidade dos indivíduos pertencerem ao setor informal ou estarem desempregados. Na comparação entre os resultados gerais com os resultados separados por sexos, não se verificou diferença marcante entre os determinantes analisados, apesar de pequenas diferenças nas magnitudes dos coeficientes.

Por outro lado, na comparação entre os sexos, foram notadas algumas diferenças importantes entre as equações: i) o fato de ser a pessoa chefe do domicílio comparado com cônjuge, filho ou outro mostrou impacto negativo para as mulheres na informalidade, uma vez que, geralmente, são as mulheres que assumem, com maior intensidade, o papel secundário no provimento da renda domiciliar, da mesma forma que, quando comparadas com os homens, assumem com mais frequência às atividades domésticas, como o fato também de mulheres casadas geralmente terem filhos, o que diminui as chances de participação nos empregos formais, em virtude de estarem mais associadas às mulheres a educação e os cuidados relativos às crianças. As mulheres acabam buscando empregos mais próximos de casa e acabam recorrendo a informalidade; ii) os efeitos marginais da escolaridade para as mulheres apresentaram-se superiores aos dos homens, indicando que as mulheres são mais sensíveis a informalidade e desemprego comparadas aos homens quando analisadas a escolaridade.

Sobre a variável escolaridade, ressalta-se que ela apresentou grande impacto sobre a probabilidade no mercado de trabalho. Nesse sentido, como sugerido por Cirino \& De Lima (2011) as politicas públicas feitas pelo governo federal deve não apenas continuar com os investimentos em educação como aumentá-los, uma vez que tais ações contribuem fortemente para a possibilidade da redução da informalidade e desemprego no mercado de trabalho. Tendo em vista os resultados encontrados, esse 
estudo é de extrema importância para o meio econômico e social, bem como contribui para novas pesquisas, visto a necessidade da implantação de novos projetos de incentivo a educação, e a continua ampliação das políticas sociais para a educação de nível superior.

\section{Referências}

BALSADI, O. Características do emprego rural no Estado de São Paulo nos anos 90. Campinas: Unicamp, 2000 (dissertação de mestrado).

BRAGA, Thaiz Silveira. "O setor informal e as formas de participação na produção": os casos das Regiões Metropolitanas de Salvador e Recife. Anais, 2016, 1-23.

CARNEIRO, F.G.; HENLEY, A. Modelling formal vs. informal employment and earnings: microeconometric evidence for Brazil. Encontro Nacional de Economia. Anais. ANPEC, 2001.

CIRINO, Jader Fernandes; DE LIMA, João Eustáquio. Determinantes da participação feminina no mercado de trabalho: uma comparação entre os sexos e entre os mercados das regiões metropolitanas de Belo Horizonte e Salvador. Revista Econômica do Nordeste, 2011, 42.1: 165-182.

CORRÊA, Carolina Rodrigues; LIMA, João Eustáquio de. Determinantes da participação e dos rendimentos dos jovens no mercado de trabalho: o caso da Região Metropolitana de Recife. Cadernos Metrópole, 2015, 17.34: 541-553.

CRANE, R. (2007) “Is There a Quiet Revolution in Women's Travel? Revisiting the Gender Gap in Commuting”, Journal of the American Planning Association, Summer 2007, 73: 298-316.

DALBERTO, C.R. Formalidade vs. Informalidade no mercado de trabalho brasileiro: uma investigação dos diferenciais de rendimento. Viçosa, MG: UFV, 2014. 87 f. Dissertação (Mestrado em Economia) Programa de Pós-Graduação em Economia da Universidade Federal de Viçosa. Viçosa, 2014.

DUARTE, Leandro Batista; CIRINO, Jader Fernandes; SETTE, Ana Beatriz Pereira. DIFERENCIAL DE SALÁRIOS NO MERCADO FORMAL/INFORMAL PARA AS REGIÕES METROPOLITANAS DO NORDESTE. Revista Econômica do Nordeste, 2018, 49.1: 9-24.

DUJARDIN, C., SELOD, H. AND THOMAS, I. (2008) Residential segregation and unemployment: the case of Brussels, Urban Studies, 45, pp. 89-113. 
FERNANDES, R. Mercado de trabalho não-regulamentado: participação relativa e diferenciais de salários. Pesquisa e Planejamento Econômico, v.26, n. 3, p. 417-442, 1996.

GOBILLON, L. AND SELOD, H. (2006). Ségrégation résidentielle, accessibilité aux emplois et chômage: le cas de l'Ile de France. Working Paper No. 0605, Laboratoire d'Economie Appliquée, INRA, Paris.

GREENE, W. Econometric analysis. 5th ed. New York: Prentice Hall, 2003. 1026 p.

HAIR, J. F., et al. Análise multivariada de dados. Trad. Adonai S. Sant'Anna e Anselmo C. Neto. 5 ed. Porto Alegre: Bookman, 2005.

HOFFMANN, R.; LEONE, E. T. Participação da mulher no mercado de trabalho e desigualdade da renda domiciliar per capita no Brasil: 1981-2002. Nova Economia, Belo Horizonte, n.14, 2004.

IBGE. Censo Demográfico 2010. Notas Técnicas. Rio de Janeiro, Instituto Brasileiro de Geografia e Estatística, 2010. IBGE. Censo Demográfico 2010, Microdados da Amostra de Domicílios. Rio de Janeiro, Instituto Brasileiro de Geografia e Estatística, 2010.

IBGE. Censo Demográfico 2010, Microdados da Amostra de Pessoas. Rio de Janeiro, Instituto Brasileiro de Geografia e Estatística, 2010.

JOHNSTON, R. J., JONES, K., BURGESS, S. et al. (2004) Scale, factor analyses, and neighborhood effects. Geographical Analysis, 36(4), pp. 350-368.

LAVINAS, L.; AMARAL, M. R.; BARROS, F. Evolução do desemprego feminino nas áreas metropolitanas. Texto para Discussão: IPEA, Rio de Janeiro, n.756, 2000.

LEE B. SONG; MACDONALD J. F. (2003). Determinants of commuting time and distance for Seoul Residents: The impact of family status on the commuting of women. Urban Studies, Vol. 40, No. 7, 1283-1302,

MACHADO, A.F.; OLIVEIRA, A.M.H.C.; ANTIGO, M. Evolução do diferencial de rendimentos entre setor formal e informal no Brasil: o papel das características não observadas. Revista de Economia Contemporânea, v. 12, n. 2, p. 355-388, 2008.

MENEZES-FILHO, N.A.; MENDES, M.; ALMEIDA, E.S. O diferencial de salários formal-informal no Brasil: segmentação ou viés de seleção? Revista Brasileira de Economia, v. 58, n. 2, p. 235-248, 2004. 
O’REGAN, K. M. QUIGLEY, J. M. (1996) Spatial effects upon employment outcomes: the case of New Jersey teenagers. New England Economic Review, May/June, pp. 41-58.

PIANTO, MARIA E. TANNURI; AND PIANTO, DONALD M.: "Informal Employment in Brazil - A Choice at the Top and Segmentation at the Bottom: A Quantile Regression Approach", Texto para discussão nº 236. Brasília, agosto de 2002.

PICCHETTI, P.; CHAHAD, J. P. A evolução da taxa de desemprego estrutural no Brasil: uma análise entre regiões e características dos trabalhadores. In: ENCONTRO NACIONAL DA ASSOCIAÇÃO NACIONAL DE PÓS-GRADUAÇÃO EM ECONOMIA, 31., João Pessoa, 2003. Anais eletrônicos... João Pessoa, 2003.

WOOLDRIDGE, J. M. Econometric analysis of cross-section and panel data. Cambridge: The MIT Press, 2002. p. 735

\section{Notas de Rodapé}

2. Base disponível até o momento.

3. Conforme a revisão atual, o município de Goiana passou a integrar a região de desenvolvimento Mata Norte e não mais a região metropolitana do Recife, que foi reduzida a 14 municípios: Abreu e Lima, Araçoiaba, Cabo de Santo Agostinho, Camaragibe, Igarassu, Ilha de Itamaracá, Ipojuca, Itapissuma, Jaboatão dos Guararapes, Moreno, Olinda, Paulista, São Lourenço da Mata e a capital pernambucana.

4. Define-se área de ponderação como sendo uma unidade geográfica, formada por um agrupamento de setores censitários, para a aplicação dos procedimentos de calibração das estimativas com as informações conhecidas para a população como um todo (IBGE, 2010).

5. Os demais municípios da RMR foram excluídos, visto que não se incluem nos critérios estabelecidos pelo Censo 2010 para apresentarem áreas de ponderação. Para o Censo Demográfico 2010, foram usados métodos e sistemas automáticos de formação de áreas de ponderação que conjugam critérios tais como tamanho (para permitir estimativas com qualidade estatística em áreas pequenas), contiguidade (no sentido de serem constituídas por conjuntos de setores limítrofes com algum sentido geográfico) e homogeneidade em relação a um conjunto de características populacionais e de infraestrutura conhecidas.

6. O IBGE realiza, a cada dez anos, um Censo Demográfico, com desagregação regional ao nível municipal (ou ao nível da área censitária dos municípios maiores). O Censo Demográfico coleta informações sobre as principais características dos indivíduos e famílias, fornecendo detalhes sobre as condições de vida da população em cada município e servindo como um instrumento de política muito importante em um país. Um questionário mais curto aplica-se a toda a população ao nível do setor censitário, enquanto as características individuais específicas são 
investigadas em um conjunto mais longo de questões que são dadas a uma amostra e são representativas ao nível das áreas ponderadas (conglomerados de setores censitários com pelo menos 400 domicílios). 